\title{
Outcome of Treated Paediatric Clinical Meningitis, With and Without Cerebro Spinal Fluid (CSF) Analysis at a Tertiary Care Hospital of Karachi
}

\author{
Sina Aziz* and Mussarat Ameer \\ Paediatric unit 2, Abbasi Shaheed Hospital and Karachi Medical and Dental College, Pakistan
}

Submission: October 17,2019; Published: November 20, 2019

*Corresponding author: Sina Aziz, Paediatric unit 2, Abbasi Shaheed Hospital and Karachi Medical and Dental College, Pakistan

\begin{abstract}
Objective: To determine the outcome of paediatric clinical meningitis with and without Cerebro spinal fluid (CSF) examination at a tertiary care hospital of Karachi.

Methods: Patients presenting at the paediatric unit 2 emergency at Abbasi Shaheed Hospital (ASH) with, clinical suspicion of meningitis from June 2013 to 31 Jan 2018 were included, regardless of whether the CSF examination was done or not. Immunization received by the children was noted. Children were followed for mortality and morbidity to detect sequelae of meningitis if present.

Results: A total of 450 children were included, 285 (63.3\%) male and 165 (36.6\%) female. Age range; 7 months to 144 months (12 years). In 235/450 (52.2\%) children CSF was not done, (due to lack of parents' consent), while in 215/450 (47.7\%) CSF was done, with culture positive in 8/215 (3.7\%). Sequelae were present in two children with CSF and two children without CSF. None of the children expired. In the eight patients with positive culture, five $(5 / 8,62.5 \%)$ children were not vaccinated.
\end{abstract}

Conclusion: With and without CSF examination did not affect the mortality. However, morbidity was present in patients, both with and without CSF examination. In patients with positive CSF culture, majority was unvaccinated.

Keywords: Meningitis; Pediatrics; Cerebrospinal fluid; Sequalae; Outcome

\section{Introduction}

Meningitis can be defined as an inflammation of arachnoid and pia matter caused by bacteria, viruses, mycobacterium tuberculosis (TB) and rarely, by fungi [1]. Bacterial meningitis is one of the most potentially serious infections occurring in infants and older children. This infection is associated with a high rate of acute complications and risk of long-term morbidity [2]. Over 1.2 million cases of bacterial meningitis are estimated to occur worldwide each year more so in the low and middle-income countries [3]. It is clinically diagnosed in the presence of following signs and symptoms, apnea, stiff or painful neck, headaches, hearing loss, fever without a source and irritable Infant [1]. Meningitis is a critical disease that needs rapid diagnosis and initiation of appropriate early treatment, otherwise it could lead to permanent disabilities or death, since there is a high rate of mortality for meningitis [4]. Mycobacterium tuberculosis is responsible for serious infections in developing countries, about 32, 000 children (less than 15 year of age), develop Multidrug resistant TB globally [5]. In a multi Centre study from Sindh, Pakistan, extrapulmonary Tuberculosis was seen in 112 cases and
22/112 (19.6\%) had tuberculosis meningitis, maximum number of which were present in children $\leq 2$ years of age6. Children who were infected as neonates have more health and developmental problems than those who had meningitis when they were older than one month. The risk of meningitis is increased among infants and young children with occult bacteremia [6,7]. The diagnosis of acute pyogenic meningitis is confirmed by analysis of the cerebrospinal fluid (CSF), which typically reveals microorganisms on Gram stain and culture, a neutrophilic pleocytosis, elevated protein, and reduced glucose concentrations. The Gram stain is positive in $70-90 \%$ of patients with untreated bacterial meningitis. CSF evaluation is the single most important aspect of the laboratory diagnosis of meningitis [8].

Hence, meningitis in pediatric population requires a clinical assessment, with lab analysis of cerebrospinal fluid, its culture and Complete blood count ( $\mathrm{CBC}$ ) and blood culture (in some cases) [1]. The rationale for doing this audit was that in our clinical practice, we encounter a high number of parents who refused to give consent for lumber puncture (LP) to be performed 
in their child with suspected meningitis, despite counseling. Our community is made up of many ethnic groups and each ethnic group has their own unique socio-cultural beliefs about LP and this could be a strong influence on their decision whether to agree or refuse LP. Also, our observation was that majority of the children coming to our tertiary care hospital had already received antibiotics and our greatest concern was that a significant number were not immunized as per requirement of the Expanded Program of Immunization (EPI) of World Health Organization (WHO) for Pakistan [9]. Keeping above viewpoints in mind, audit was conducted to evaluate the common organism in children presenting at Abbasi Shaheed hospital (ASH) a tertiary care hospital, with paediatric clinical meningitis (fits, fever, irritability, reluctance to feed, lethargy and/or bulging fontanel) and to determine the outcome of children treated with meningitis, with and without a CSF analysis, with same treatment being given to both type of patients, during admission as per standard protocols.

\section{Methods}

Table 1: Expanded Program of Immunization of Pakistan in children.

\begin{tabular}{|c|c|c|c|}
\hline When & Age & \multicolumn{2}{|c|}{ Vaccine } \\
\hline At birth & At birth & BCG & OPV, Hepatitis B \\
\hline $2^{\text {nd }}$ Visit & 6 weeks & OPV1, *Pneumococcal 1 & $\begin{array}{c}\text { Rotavirus } 1 \\
\text { **Pentavalent } 1\end{array}$ \\
\hline $3^{\text {rd }}$ Visit & 10 weeks & OPV2, *Pneumococcal 2 & $\begin{array}{c}\text { Rotavirus } 2, \\
\text { **Pentavalent } 2\end{array}$ \\
\hline $4^{\text {th }}$ Visit & 14 weeks & OPV3, *Pneumococcal 3 & IPV, **Pentavalent 3 \\
\hline $5^{\text {th }}$ Visit & 9 months & Measles 1 & \\
\hline $6^{\text {th }}$ Visit & 15 months & Measles 2 & \\
\hline
\end{tabular}

BCG-Bacillus Calmette Guerin, OPV-Oral Polio Vaccine, IPV-Injectable Polio Vaccine

*Vaccine covers meningitis infection secondary to pneumococcus.

**Pentavalent vaccine includes Diphtheria, Pertussis, Tetanus, Hepatitis $\mathrm{B}$ and $H$. Influenza.

(http://www.epi.gov.pk/immunisation-schedule/EPI Pakistan, accessed on June 17, 2019) [9].

All paediatric patients presenting at the paediatric unit 2 emergency of ASH with age above one month to 144 months (12 years of age) and clinical suspicion of meningitis or referred to ASH, for treatment of meningitis with and without cerebrospinal fluid (CSF) analysis were included in this study, from June 2013 to 31 Jan 2018. Parents did not always agree to CSF by lumber puncture, despite counseling. These children were also included in the study due to strong clinical suspicion of meningitis. Children with clinical manifestations (signs and symptoms: fits, fever, irritability, reluctance to feed, lethargy and/or bulging fontanel), of meningitis, whether specific or non-specific were included [1]. Those children with suspected febrile seizures which could not be excluded with certainty were also included in this study with the protocol of suspected meningitis. Those patients referred to another hospital, (either due to parents wish or sick patients which required a ventilator, and this was not available at ASH) and patients who left against medical advice (LAMA) at any stage have been excluded. All patients received standard protocol of treatment [1]. The immunization schedule of the children (Table 1) was noted, according to the Expanded program of Immunization (EPI) of Pakistan [9]. Presence of an immunization card for verification was essential; absence of the card and only history from the parents was considered incomplete immunization. For Tuberculosis, children were labeled as vaccinated with presence of BCG scar and unvaccinated without BCG scar, regardless of the history of vaccination. The children had a complete blood picture (CP) with peripheral smear, random blood sugar (RBS), CSF for DR and cultures (CS) including CS for Acid Fast Bacilli (AFB) (if parents agreed for lumber puncture for complete CSF analysis), blood culture, UCE (urea, creatinine and electrolytes) and other tests if required. Children were treated for meningitis with the standard protocol1. Viral studies for meningitis were not available at our institute. In case the child did not respond to intravenous antibiotics within $48 \mathrm{hrs}$ or a clinical suspicion of viral meningitis or encephalitis was present, acyclovir was added, and further management changed depending upon the CSF DR and culture report [1]. In the ward and following discharge, the patient was followed at the outpatient department to detect mortality and morbidity (presence of any sequelae) of meningitis if present.

Results

Table 2: Age distribution of children with clinical meningitis $(n=450)$.

\begin{tabular}{|c|c|c|}
\hline Age & $\mathbf{N}(\%)$ & Mean \pm SD (age in months) \\
\hline 1 month & $7(1.5)$ & - \\
\hline >1 month-1 year & $213(47.3)$ & $19.36 \pm 8.75$ \\
\hline >1 year-5 year & $193(42.8)$ & $4.11 \pm 9.4$ \\
\hline >5-year-12 year & $37(8.2)$ & $0.4 \pm 1.7$ \\
\hline
\end{tabular}

A total of 450 children were included, 285 (63.3\%) male and $165(36.6 \%)$ female. One month of age were $7(1.5 \%),>1$ month to one year $213(47.8 \%)$, >one year to 5 year $189(42.4 \%)$ and $>5$ to 12 year 37 (8.3\%), (Table 2). A total of 235/450 (52.2\%) children did not have a CSF done, while in 215/450 (47.7\%) a CSF was done. In the latter group with CSF examination done, cultures with positive CSF were 8/215 (3.7\%) with details of the organisms isolated as shown in (Table 3). By history; children vaccinated with BCG scar were 245 (54.3\%), vaccinated but BCG scar absent were 62 (13.7\%), while unvaccinated were 143 (31.7\%). In the eight patients with positive culture, five $(5 / 8, \%)$ children were not vaccinated, and BCG scar was absent. Regarding mortality, none of the children expired. While in terms of morbidity, children, without CSF, 2 had sequelae, of which, one had hemiplegia, who on follow up responded with treatment and did not have further sequalae, while the other on MRI showed a tuberculoma, with treatment the child responded and had no deafness or speech or motor deficit, when followed at the paediatric OPD. In children with CSF examination, 2 patients had sequalae one was a 2 year old female child with streptococcus pneumonia on culture of CSF, parents did not complete treatment and left against medical advice (LAMA) and on follow up at OPD, child developed hydrocephalus was referred to neurosurgery for further management. The 2nd 
was a male, child, CSF showed, only presence of RBC and MRI done showed acute ischemic infarcts. The ventricular system, basal cistern and cortical sulci were dilated. This child had sequelae even when followed at the OPD he was unable to sit and there was decreased limb movement. None of the children expired (Figure 1). These sequelae were present even in the ward while the children were on treatment and it accounted for $0.8 \%$ of the total 450 children admitted with meningitis.

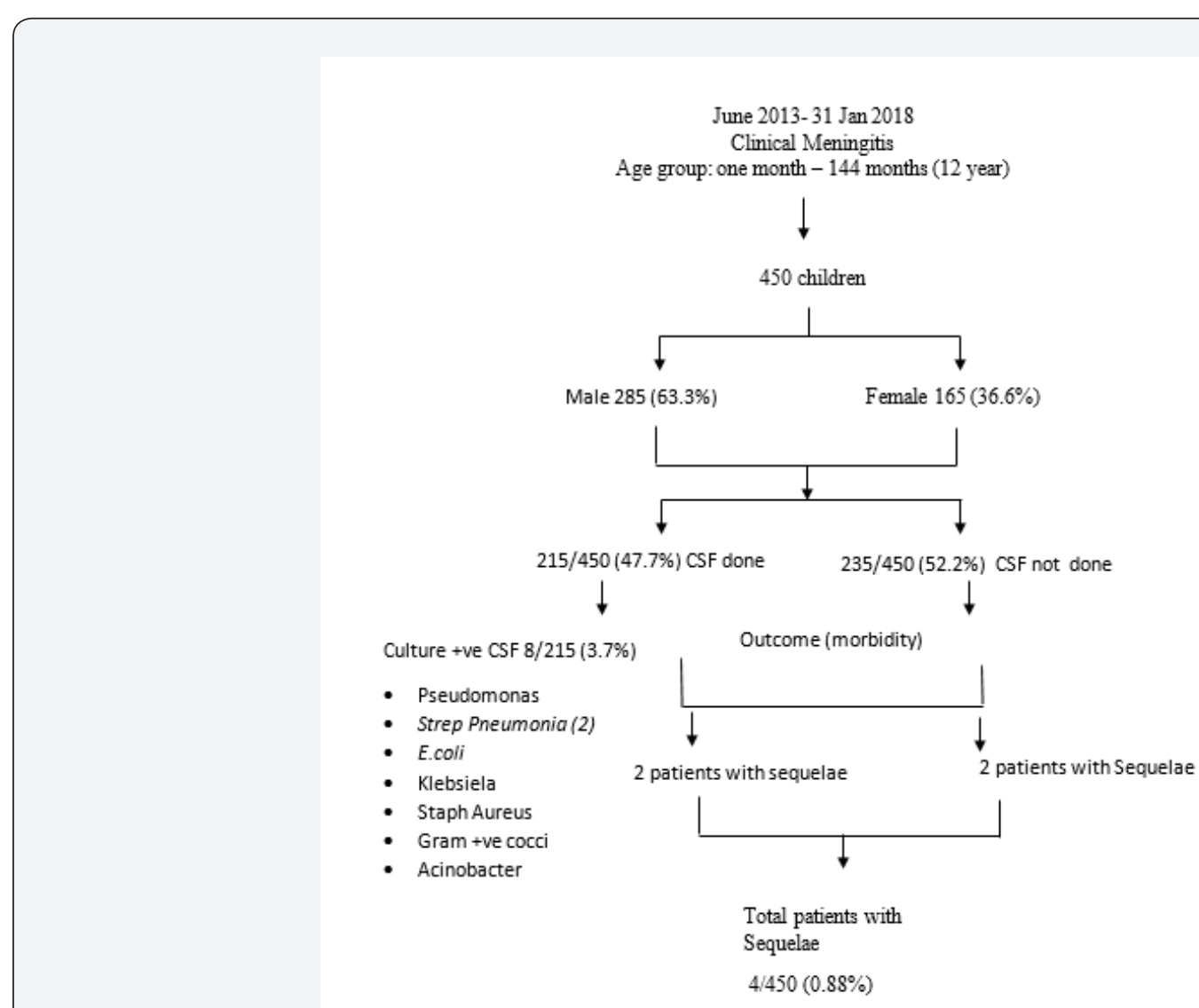

Figure 1

Table 3: Children (age in months) with meningitis and culture positive cerebrospinal fluid (CSF) at a tertiary care hospital of Karachi.

\begin{tabular}{|c|c|c|c|c|c|c|c|c|c|c|c|c|}
\hline S. no & Age & Sex & BCG & RBS & \multicolumn{7}{c|}{ CSF Detailed Report (DR) } & *CSF culture \\
\hline \multicolumn{9}{|c|}{} & \multicolumn{9}{|c|}{} & Glucosemg/dL & Proteinmg/dL & TLC/cumm & TLCN\% & TLCL\% & RBC/cumm & \\
\hline 1 & 9 & M & yes & 100 & 45 & 300 & 40 & 60 & 40 & nil & Pseudomonas aeruginosa \\
\hline 2 & 7 & F & yes & 97 & 42 & 256 & 25 & 40 & 60 & nil & Streptococcus pneumonia \\
\hline 3 & 42 & F & no & 101 & 50 & 154 & 5 & & & 2 & E. coli \\
\hline 4 & 11 & M & no & 80 & 10 & 152 & 1900 & 82 & 10 & 600 & Streptococcus pneumonia \\
\hline 5 & 1 & F & no & 75 & 19 & 563 & 70 & 60 & 40 & nil & Klebsiella species \\
\hline 6 & 10 & M & yes & 119 & 60 & 12 & 2 & - & - & 6 & Staphylococcus aureus \\
\hline 7 & 132 & F & no & 70 & 5 & 133 & 19200 & 90 & 10 & 25 & Gram positive cocci \\
\hline 8 & 12 & M & no & 136 & 74 & 100 & 3 & - & - & - & Acinobacter species \\
\hline
\end{tabular}

\section{Discussion}

A total of 450 cases of suspected meningitis were included. Only $48 \%$ had CSF analysis. Outcome of these patients with and without CSF has been determined in this paper. Also, immunization status of the children in this study is discussed, as majority of the bugs causing meningitis, (Streptococcus pneumonia, Tuberculosis, H. influenza, measles virus, E. coli in neonates) are covered by EPI of Pakistan [9], and despite freely available immunization programs in the country majority of children were not immunized, as also documented by previously published data of the region $[10,11]$. The world Health organization in 2017, has estimated 19.9 million infants worldwide did not receive, routine immunization services such as 3 doses of DTP vaccine. Around $60 \%$ of these children live in 10 countries: Afghanistan, Angola, the Democratic Republic of the Congo, Ethiopia, India, Indonesia, Iraq, Nigeria, Pakistan and South Africa [11]. This worrisome situation is supported by the 
present data and that published in recent years locally [10]. Hence, despite counseling when parents of children with suspected clinical meningitis, refused lumber puncture for CSF analysis; $235 / 450$ (52.2\%), pediatricians had no option but to treat the patient with clinical suspicion of meningitis for 10 days with intravenous antibiotics as per standard protocol1. These children were routinely followed, and this audit was done to determine the outcome, in terms of morbidity i.e. sequelae (present in the child while admitted and under treatment or on follow up at the outpatient department) and mortality; death of a patient with meningitis. Two patients had sequelae both, in the group with and without CSF examination (0.8\%), indirectly suggesting whether a warrant for CSF examination is justified in each child and whether we as pediatricians are scared for a worst outcome and rightly so as sequalae though in few patients, but have been documented in published literature [12] following meningitis.

Lack of gram stain and culture positive CSF, of patients was seen in this audit, as supported by previous published data [13] with suspected bacterial meningitis. This could also be explained by the fact that majority of the children presenting at our hospital, had received antibiotic14, hence it is difficult to exclude partially treated meningitis. Partially treated meningitis, refers to bacterial meningitis complicated by antibiotic treatment before the lumbar puncture, which may result in negative CSF cultures, although other CSF findings suggestive of bacterial infection persist. The aetiology can sometimes be confirmed by polymerase chain reaction of the CSF. Majority of the local practitioners tend to use cephalosporins injectable or oral and even drugs such as linezolid without scientific evidence, as first line therapy for patients presenting to them with fever especially if the child appears to be sick or the parents are overanxious or without a valid clinical scientific reason. Latter is based on an observation while working in a government hospital and is not supported by any published data, so can be biased. Due to the partial treatment, the CSF done in our $215 / 450(47.7 \%)$ showed only $8 / 215$ (3.7\%) patients had a culture proven meningitis. Hence, remaining could be considered as partially treated, in support of previous published data $[13,14]$. Majority of those in which CSF was not done had also received antibiotics prior to coming to our hospital. In the eight patients with positive culture, five $(5 / 8,62.5 \%)$ children were not vaccinated and BCG scar was absent. This could indicate the like hood of the child acquiring meningitis and other preventable infections covered by EPI of Pakistan, due to lack of immunization $[9,10,11]$. Organism isolated in these cases according to age in months was: E.coli (42 months), streptococcus pneumonia two cases ( 7 and 11 month), klebsiella (1 month), gram-positive cocci (132 months) and acinobacter species (12 months). Most of the children were sick and admitted in PICU (paediatric intensive care unit) hence, difficult to co-relate whether the bug (other than streptococcus pneumonia) actually caused meningitis in these patients.

In this study we included children with or without CSF analysis, with febrile seizure also, especially those who were less than one year of age and/or were sick and /or whom there was even a slight clinical suspicion of meningitis. Other published guidelines [1] and data $[15,16]$ supports this approach also. It was difficult to evaluate the role of immunization in children to prevent meningitis as most of our children had incomplete immunization. It was only by history from parents that the immunization status was verified as very few had the immunization card available. Majority were immunized for tuberculosis as they had the BCG scar 245 (54.3\%) supported by previous data [10,11] 62(13.7\%) gave a history of being vaccinated but BCG scar was absent and $143(31.7 \%)$ said that immunization had not been done. This highlights the seriousness of the situation that the vaccination card was not present in majority and only by history and/or by the BCG scar, verification of immunization status was done. Even if the BCG scar was present this did not include later immunization confirmations of 2 nd visit ( 6 week old), 3rd visit (10 week old), 4 rth visit (14 week old), 5th visit ( 9 month old) and 6th visit (15 month old) as recommended for our region by WHO9,11 (Table $3)$. The incomplete immunization will lead to various diseases in our country and the problem needs to be confronted by health policy makers for further improvement. In this research, it was difficult to determine prior antibiotic exposure duration and length of illness, prior to presentation. The majority of children in our setting may not have had meningitis; hence early diagnosis of the actual cause at a local practitioner level could reduce the risk of eventual costly treatment. Also, there is no agreement as to what comprises a genuine risk of meningitis to justify a CSF in the absence of sufficient evidence. Published literature supports our observations and suggests a risk of 1 in 12 risk if the diagnosis of meningitis is missed or is delayed [17]. Despite efforts taken by WHO to publish material for childcare and health especially for developing world e.g. Integrated management of childhood illness, (IMCI) [18], health care of children is not being done as advised. For e.g. immunization is freely available in all government hospitals, children are still not being immunized. IMCI is freely available online and is not being followed by individuals involved in child health services. As a result of lack of immunization and cultural barriers childhood morbidity and mortality is affected adversely. Limitations in this research include the difficulty in evaluating the role of immunization in these children to prevent meningitis as most of the children had incomplete immunization, according to the EPI schedule of Pakistan (Table1). However, majority were presumably immunized for tuberculosis as they had the BCG scar. In countries such as Pakistan and other developing nations, 1.5 million deaths can be prevented, if global vaccination coverage improves [11]. Another limitation includes that not all the patients in whom CSF was done had viral cultures done and there was limited antigen testing and PCR for CSF, due to the tests being expensive and/or lack of availability of the specific tests, other than a few tests in the specialized labs available in Pakistan (majority of the tests done in this unit were free of cost and supported by donations). Also, a few cultures positive could be a contamination such as gram-positive cocci and acinobacter, but since the patients were in the PICU and we did not want to take a 
risk, complete treatment was given, based on our clinical acumen.

\section{References}

1. (2016) Acute bacterial meningitis beyond the neonatal period. Elsevier $20^{\text {th }}$ Edn 2940-2948.

2. Jan MM (2012) Meningitis and encephalitis in infants and children. Saudi Med J 33(1): 11-16.

3. Lukšić I, Mulić R, Falconer R, Orban M, Sidhu S, et al. (2013) Estimating global and regional morbidity from acute bacterial meningitis in children: assessment of the evidence. Croat Med J 54(6): 510-518.

4. Hasbun R (2019) Update and advances in community acquired bacterial meningitis. Curr Opin Infect Dis 32(3): 233-238.

5. Jenkins HE, Tolman AW, Yuen CM, Parr JB, Keshavjee S, et al. (2014) Incidence of multi- drug-resistant tuberculosis disease in children: systematic review and global estimates. Lancet 383(9928): 1572-1579.

6. Laghari M, Sulaiman SAS, Khan AH, Memon N (2019) A prospective study of socio-demographic, clinical characteristics and treatment outcomes of children with tuberculosis in Sindh, Pakistan. BMC Infect Dis 19(1): 82 .

7. Ali M, Chang BA, Johnson KW, Morris SK (2018) Incidence and aetiology of bacterial meningitis among children aged 1-59 months in South Asia: systematic review and meta-analysis. Vaccine 36(39): 5846-5857.

8. Nour M, Alaidarous A (2018) Clinical usefulness and accuracy of polymerase chain reaction in the detection of bacterial meningitis agents in pediatric cerebrospinal fluid. Curr Res Transl Med 66(1): 15-18.

9. (2019) Immunization schedule-Expanded Program on immunization, Pakistan.
10. Siddiqui S, Akbar M, Mehtab A, Zafar M, Shahid S, et al. (2017) Frequency of Immunisation of Children upto Five Years of Age According to Vaccination Card in Karachi - A Multicentre Study. Annals ASH KM\&DC 22(1): $12-19$

11. (2019) World Health Organization. Immunization coverage.

12. Khowaja AR, Mohiuddin S, Cohen AL, Khalid A, Mehmood U, et al. (2013) Mortality and neurodevelopmental outcomes of acute bacterial meningitis in childrenaged $<5$ years in Pakistan. J Pediatr 163(1): 8691.

13. Ku LC, Boggess KA, Cohen-Wolkowiez M (2015) Bacterial meningitis in infants. Clin Perinatol 42(1):29-45.

14. Lewin EB (1974) Partially Treated Meningitis. Am J Dis Child 128(2): 145-147.

15. Son YY, Kim GH, Byeon JH, Eun SH, Eun BL (2018) Need for Lumbar Puncture in Children Younger Than 12 Months Presenting With Simple Febrile Seizure. Pediatr Emerg Care 34(3): 212-215.

16. Bhansali P, Wiedermann BL, Pastor W, McMillan J, Shah N (2015) Management of Hospitalized Febrile Neonates Without CSF Analysis: A Study of US Pediatric Hospitals. Hosp Pediatr 5(10): 528-533.

17. Bhansali P, Wiedermann BL, Pastor W, McMillan J, Shah N (2015) Management of Hospitalized Febrile Neonates Without CSF Analysis: A Study of US Pediatric Hospitals. Hospital Pediatrics 5(10): 528-533.

18. Tadesse BT, Foster BA, Shibeshi MS, Dangiso HT (2017) Empiric Treatment of Acute Meningitis Syndrome in a Resource-Limited Setting: Clinical Outcomes and Predictors of Survival or Death. Ethiop J Health Sci 27(6): 581-588.

\section{Your next submission with Juniper Publishers will reach you the below assets}

- Quality Editorial service

- Swift Peer Review

- Reprints availability

- E-prints Service

- Manuscript Podcast for convenient understanding

- Global attainment for your research

- Manuscript accessibility in different formats

( Pdf, E-pub, Full Text, Audio)

- Unceasing customer service

Track the below URL for one-step submission https://juniperpublishers.com/online-submission.php 